\title{
Role of post-mortem computed tomography (PMCT) in the assessment of the challenging diagnosis of pericardial tamponade as cause of death in cases with hemopericardium
}

\author{
Laura Filograna $\cdot$ Patrick Laberke $\cdot$ \\ Garyfalia Ampanozi - Wolf Schweitzer • \\ Michael J. Thali $\cdot$ Lorenzo Bonomo
}

Received: 2 October 2014 / Accepted: 29 January 2015 / Published online: 19 February 2015

(C) Italian Society of Medical Radiology 2015

\begin{abstract}
Objectives When hemopericardium (HP) is found at autopsy, it represents a challenge for the forensic pathologist when having to assess its role in causing death. In fact, a proper diagnosis of pericardial tamponade (PT) must be based on clinical and instrumental data, which are not often available at post-mortem investigation. The aim of this study was to individuate post-mortem (PMCT) findings indicative for the diagnosis of PT.

Materials and methods We revised PMCT images and autopsy reports of 14 cases with fatal HP and intact pericardium. From autopsy reports, we obtained volume and cause of HP. PMCT images were reviewed to describe appearance of HP and the presence of indirect signs of increased intrapericardial pressure. A control group of 11 cases submitted to PMCT prior to autopsy was selected with the following criteria: absence of relevant pericardial effusion, venous system congestion and bleeding.

Results Of the 14 PT subjects, 13 had a double-concentric stratification of HP and compression of the coronary sinus and/or of the pulmonary trunk, all showing a flattening of the anterior surface of the heart; other findings indicative of venous system congestion were variably observed. In the control group, none of these findings was identified, with
\end{abstract}

L. Filograna $(\bowtie) \cdot$ L. Bonomo

Department of Radiological Sciences, Catholic University of Rome, School of Medicine, University Hospital "A. Gemelli", Largo A. Gemelli 8, 00168, Rome, Italy

e-mail: laura.filograna@rm.unicatt.it

L. Filograna · P. Laberke · G. Ampanozi · W. Schweitzer · M. J. Thali

Department of Forensic Medicine and Imaging, Institute of Forensic Medicine, University of Zurich, Winterthurerstrasse 190/52, 8057 Zurich, Switzerland the exception of a distended or non-completely collapsed superior vena cava (11/11 cases).

Conclusions PMCT is able to provide some findings indicative of PT. Based on this evidence, in other instances HP could be judged circumstantial rather than fatal. This study suggests the possibility to use PMCT findings to retrospectively demonstrate a clinical condition, such as PT.

Keywords Post-mortem CT · Hemopericardium · Pericardial tamponade $\cdot$ Cardiac tamponade $\cdot$ Forensic radiology $\cdot$ Forensic pathology

\section{Introduction}

Hemopericardium (HP), the pathological condition of blood in the pericardial sack, is frequently observed at autopsy. HP may originate when an intrapericardial vessel or the walls of heart chambers rupture in the pericardial sack, spontaneously or due to an injury. HP, however, can be fatal or non-fatal and the problem is to differentiate the two. Thus, when HP is detected at autopsy, it represents a challenge for the forensic pathologist who has to determine the cause of death $[1,2]$. In fact, HP results in the life-threatening condition of pericardial tamponade (PT) only when the accumulation of blood causes intrapericardial pressure to exceed the cardiac chamber diastolic pressure, thus preventing and impairing cardiac filling. Therefore, the diagnosis of PT is not based solely on the presence of HP and requires additional signs and symptoms and the support of instrumental data. Symptoms of PT include, but are not limited to, dyspnoea, tachypnea and fatigue, while common signs include tachycardia, jugular venous distension, a quiet precordium, hypotension and pulsus paradoxus (inspiratory drop in systolic blood pressure of $10 \%$ 
or $10 \mathrm{mmHg}$ ). Echocardiography is the primary clinical diagnostic method for the initial detection of pericardial effusion and can be rapidly carried out at the bedside [3, 4]; the diagnosis of PT is based on one or more bimodes and Doppler echocardiography diagnostic criteria [3, 4].

Computed tomography (CT), cardiac magnetic resonance imaging (MRI) $[3,5]$ and fluoroscopic imaging are not recommended for diagnosing PT, mainly because they are time-consuming. Nevertheless, in some cases a CT scanning performed for other purposes may detect the presence of a pericardial effusion.

The clinical literature reports some in vivo CT findings in cases of imminent PT [5-15]. They include pericardial effusion, flattened heart sign (FHS), bowing of the interventricular septum, compression of the coronary sinus and of the pulmonary trunk, dilatation of the superior vena cava (SVC) (with a diameter similar to or greater than that of the adjacent thoracic aorta) and of the inferior vena cava (IVC) (with a diameter greater than twice that of the adjacent abdominal aorta), distension of the hepatic and renal veins, periportal oedema and reflux of contrast into the azygos vein and IVC. All these CT findings are related to the effects of the increased intrapericardial pressure on the intrapericardial vessels and the cardiac chambers (compression), and on the venous vascular system (distension) and to the obstacle to the progression of the blood and consequently the contrast media towards the cardiac chambers. Although these in vivo CT signs, when seen individually, cannot be considered specific for PT, their simultaneous evidence, particularly in the presence of a large pericardial effusion, strongly suggests a diagnosis of an imminent PT [15].

In the field of forensics, while the autopsy detection of HP is quite frequent, clinical and instrumental data in the ante-mortem period are only rarely available to differentiate between fatal and non-fatal HP. In addition, the forensic literature is unclear about the minimal amount of pericardial blood required to increase intrapericardial pressure to cause death. Two hundred millilitres of clotted blood has been proposed [2], but amounts up to $300-400 \mathrm{ml}$ are generally measured at autopsies, with some authors reporting even greater volumes [16, 17]. Moreover, in trauma cases, the presence of a pericardial defect should be considered because, even after fatal HP, a perimortally acquired tear may facilitate a postmortem drainage of blood from the pericardium, particularly if intensive resuscitation manoeuvres have been performed.

Thus, when HP is found at autopsy, it does not necessarily constitute the primary cause of death.

Few studies in the forensic literature were focused on the use of PMCT imaging in cases of HP [18-24] and even fewer on the possibility to use post-mortem imaging for the assessment of the diagnosis of PT due to HP [18, 23, 24].

This work focused on autopsy cases with a clear, based on ante-mortem clinical and/or instrumental records, diagnosis of PT, submitted to PMCT whole-body scanning prior to autopsy. The aim was to individuate which PMCT finding can be considered as indicative for the diagnosis of PT (that is, for fatal HP) and, thus, to create a reference for other forensic cases where ante-mortem data are not available and other pathological conditions may have caused or concurred to cause the death.

\section{Materials and methods}

The study was approved by both our institutional review board and the public prosecution department. Post-mortem whole body CT data sets from 14 human bodies, who had been delivered to our institute for forensic evaluation including autopsy, were retrospectively selected. Inclusion criteria were PT due to acute HP as the autopsy cause of death (assessed on the basis of perimortem clinical/instrumental data) and an intact pericardium. The exclusion criteria were the presence of other internal and/or external haemorrhage, presence of concurrent causes of death, PMCT signs of decomposition (i.e. intravenous gas).

The study population consisted of eight females and six males (mean age 57 years, range $34-78$ years). In all cases, HP showed a volume superior to $150 \mathrm{ml}$. In 13/14 cases, the cause of the HP was a ruptured (dissected/aneurismal/ normal) ascending aorta. In one case, HP was caused by iatrogenic rupture of the superior vena cava, during the insertion of a catheter.

In all the cases, PMCT scanning was performed prior to autopsy, on a 128-slice Somatom Definition Flash Dual Source CT scanner (Siemens Healthcare, Forchheim, Germany), with the following parameters: $120 \mathrm{kV}, 350-1,000$ $\mathrm{mAs}$ dependent on automatic exposure control with tube current-time modulation (CareDose 4D, Siemens Healthcare, Forchheim, Germany), $128 \times 0.6 \mathrm{~mm}$ collimation, $0.5 \mathrm{~s}$ rotation and 0.6 pitch. Image reconstruction was carried out using a soft and hard tissue convolution kernel with a slice thickness of $1 \mathrm{~mm}$ and an increment of $0.6 \mathrm{~mm}$, in an abdominal and lung window. All the bodies were scanned within $48 \mathrm{~h}$ since the estimated time of death.

All image data sets were interpreted using a Sectra Workstation IDS7 (version 14.3.5.136, Sectra, Linkoping, Sweden), to describe the PMCT appearance of the HP, with particular attention being paid to hyperdense armoured heart (HAH), the PMCT sign described by Shiotani et al. [20], and to annotate indirect CT signs of an increased intrapericardial pressure, as they are reported in the clinical literature [5-15]. In particular, the presence of the following CT findings was assessed: FHS, distension of hepatic and/or renal veins, compression of the coronary sinus and/or of the pulmonary trunk, periportal oedema. About the distension of the SVC and IVC, performing a comparison between the diameters of 
Table 1 Results of PMCT imaging analysis in the study group

\begin{tabular}{|c|c|c|c|c|c|c|c|c|c|}
\hline Case no. & HP features & FHS & $\uparrow \varnothing \mathrm{SVC}$ & $\uparrow \varnothing \mathrm{IVC}$ & $\begin{array}{l}\uparrow \varnothing \text { Hepatic } \\
\text { veins }\end{array}$ & $\begin{array}{l}\uparrow \varnothing \text { Renal } \\
\text { veins }\end{array}$ & $\begin{array}{l}\downarrow \varnothing \text { Coronary } \\
\text { sinus }\end{array}$ & $\begin{array}{l}\downarrow \varnothing \text { Pulmonary } \\
\text { trunk }\end{array}$ & $\begin{array}{l}\text { Periportal } \\
\text { oedema }\end{array}$ \\
\hline 1. & HAH & + & \pm & - & - & - & + & + & \pm \\
\hline 2. & HAH & + & \pm & - & \pm & \pm & + & + & - \\
\hline 3. & HAH & \pm & \pm & - & - & \pm & + & + & \pm \\
\hline 4. & HAH & \pm & \pm & - & - & - & + & + & - \\
\hline 5. & HAH & + & \pm & \pm & \pm & - & + & + & - \\
\hline 6. & НАH & \pm & \pm & \pm & \pm & - & + & + & - \\
\hline 7. & НАH & + & \pm & \pm & - & + & + & + & - \\
\hline 8. & HAH & \pm & \pm & \pm & \pm & + & + & + & + \\
\hline 9. & HAH & \pm & \pm & \pm & - & + & + & + & - \\
\hline 10. & HAH & \pm & \pm & \pm & - & \pm & + & + & - \\
\hline 11. & НАН & + & \pm & \pm & + & \pm & - & \pm & - \\
\hline 12. & FFL & \pm & \pm & - & \pm & \pm & - & \pm & - \\
\hline 13. & НАН & \pm & \pm & \pm & - & \pm & + & + & - \\
\hline 14. & HAH & + & \pm & \pm & - & + & + & + & - \\
\hline
\end{tabular}

$H P$ hemopericardium, $H A H$ hyperdense armoured heart, $F F L$ fluid-fluid level, $F H S$ flattened heart sign, $S V C$ superior vena cava, $I V C$ inferior vena cava

- Finding absent, + finding markedly present, \pm finding not markedly present

these vessels and that of the ascending and abdominal aorta, as proposed by the clinical literature, was not possible in many cases, because of the presence of an aortic aneurysm, a deformity of the profile of the aorta or the collapse of the aorta caused by dissection and/or rupture of the vessel, or due to the usual, progressive collapsing of the vessels in the post-mortem period [25]. Thus, in every case, it was annotated only the presence or absence of almost complete collapse of SVC and IVC. Other signs, such as the angulation or bowing of the interventricular septum, and other findings related to reflux of contrast material were excluded because contrast injection was not performed and a reflux of contrast is not documentable on PMCT angiography.

A control group of 11 non-PT cases submitted to PMCT prior to autopsy was retrospectively selected with the following macroscopic criteria: an intact pericardium, absence of relevant pericardial effusion on autopsy reports, venous system congestion and bleeding.

A preferential research was conducted between deaths caused by intoxication, and suffocation related to smothering, choking, or reduction of the oxygen concentration in the respired atmosphere, as it occurs with $\mathrm{CO}$ intoxication, or when a plastic bag is placed over the head down to neck level. Cases with PMCT signs of decomposition (i.e. intravenous gas) were excluded. The control group population consisted of five females and six males (mean age 46 years, range 3-80 years). All the bodies were scanned with the same parameters of the study group, within $48 \mathrm{~h}$ since the estimated time of death.
The same indirect CT signs of an increased intrapericardial pressure were investigated also in the PMCT data sets related to the control group.

\section{Results}

Study group

The results of the retrospective PMCT imaging analysis are summarized in the Table 1.

Of the 14 subjects with autopsy-ascertained death due to PT, all cases showed a stratification of the intrapericardial blood: 13 showed HAH of variable thickness and homogeneity in thickness (Fig. 1), and one case (no. 12) showed a fluid-fluid level. All cases showed FHS of variable entity (Fig. 2) and compression of the coronary sinus and/or of the pulmonary trunk (Fig. 3). Other findings indicative of venous system congestion were variably observed on PMCT. In particular, hepatic and/or renal veins distension were observed in 12/14 cases (Fig. 4). A complete collapse of the SVC was never observed, and the IVC in the infrahepatic tract appeared not completely collapsed in 9/14 cases (Fig. 5). Finally, some periportal oedema was observed in 3/14 cases.

\section{Control group}

The results of the retrospective PMCT imaging analysis are summarized in the Table 2. 

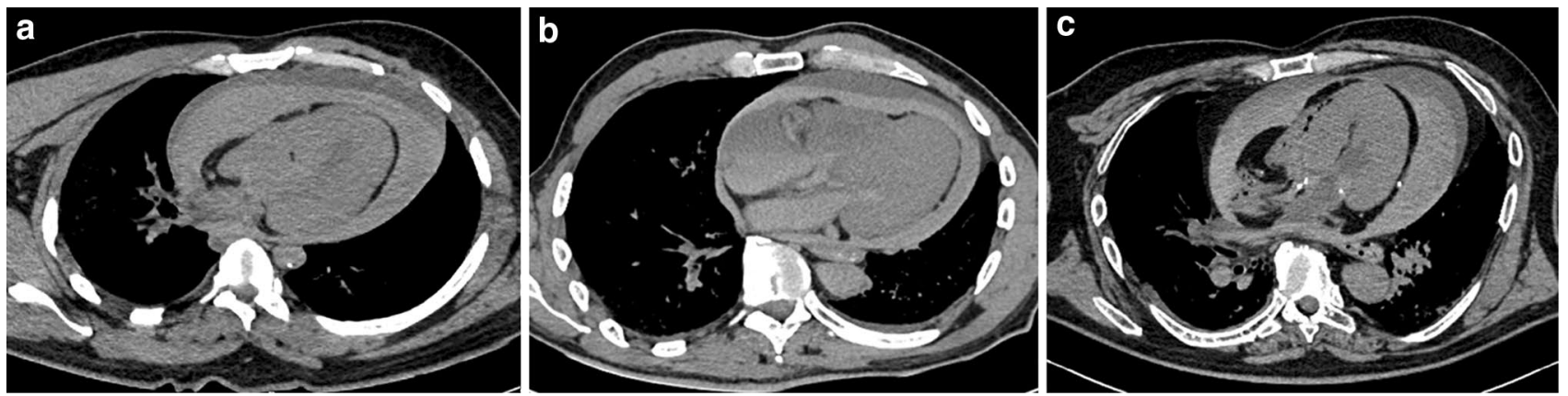

Fig. 1 The CT axial images $(\mathbf{a}, \mathbf{b}, \mathbf{c})$ at the cardiac level, referred to three cases of the study group show a double density stratification of the hemopericardium. The images $\mathbf{a}$ and $\mathbf{b}$ show the inner ring homo-

geneous in thickness, but thinner in (b) than in (a). In the image $\mathbf{c}$, the inner densest ring appears dis-homogeneous in thickness
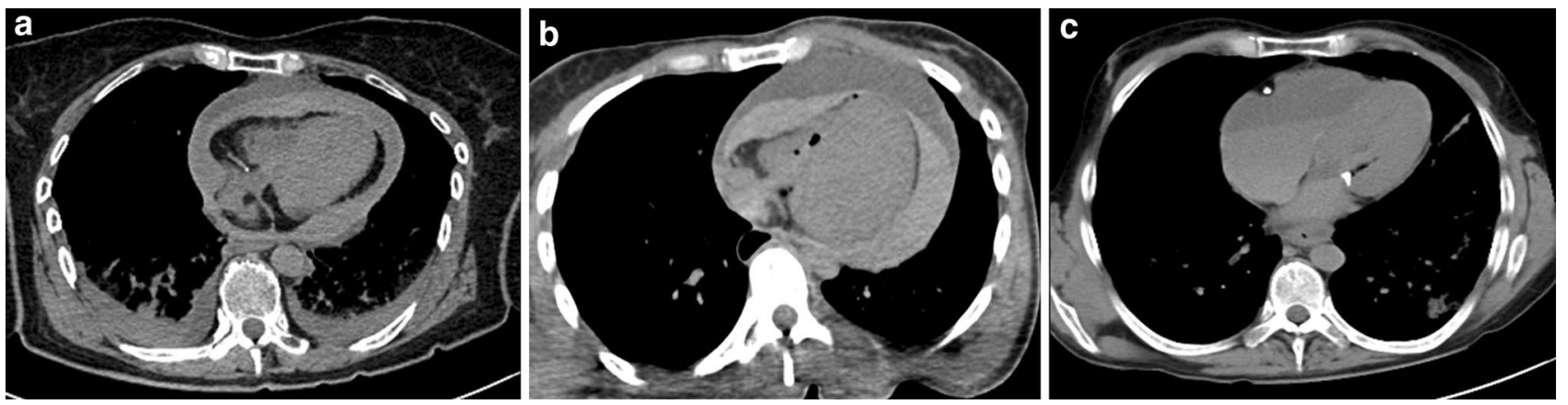

Fig. 2 The CT axial images $(\mathbf{a}, \mathbf{b}, \mathbf{c})$ at the cardiac level are referred to two cases of the study group $(\mathbf{a}, \mathbf{b})$ and a case of the control group (c). The images $\mathbf{a}$ and $\mathbf{b}$ show the flattening of the anterior surface of the right chambers (FHS), more pronounced in (b) with respect to (a). The image $\mathbf{c}$ of a case of the control group shows the absence of FHS
Fig. 3 The CT axial images $(\mathbf{a}, \mathbf{b}, \mathbf{c}, \mathbf{d})$ at the cardiac level are referred to two cases of the study group $(\mathbf{a}, \mathbf{b})$ and to two cases of the control group $(\mathbf{c}, \mathbf{d})$. In $\mathbf{a}$ and $\mathbf{b}$, the compression of the coronary sinus (a) and of the coronary trunk (b) are shown. In $\mathbf{c}$ and $\mathbf{d}$, the coronary sinus (c) and the pulmonary trunk (d) appear not compressed
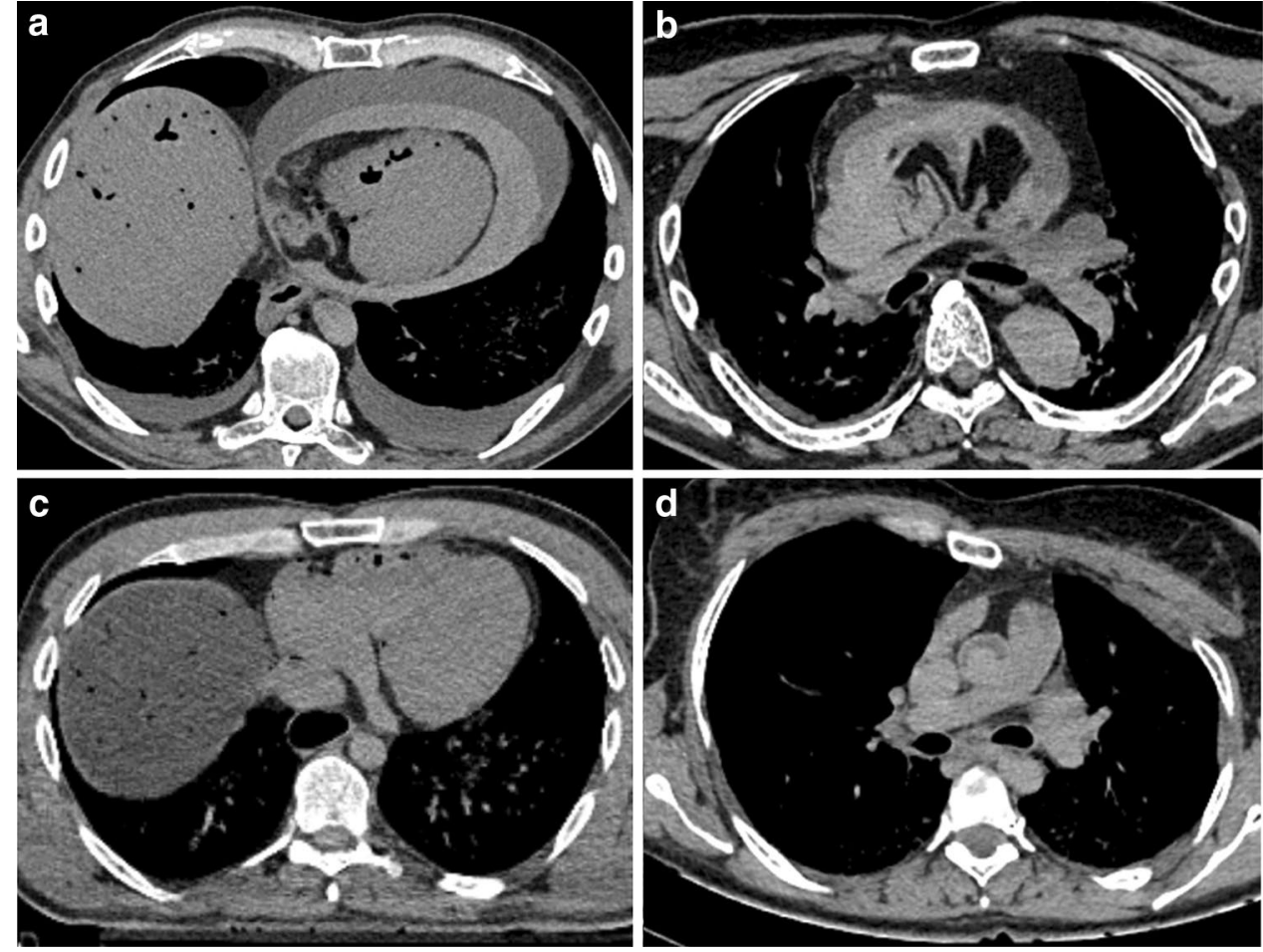
Fig. 4 The CT axial images at the hepatic $(\mathbf{a}, \mathbf{c})$ and renal (b, d) veins level are referred to two cases of the study group (a, b) and to two cases of the control group (c, d). The images a and $\mathbf{b}$ show the distension of the hepatic and renal veins, whereas in the images $\mathbf{c}$ and $\mathbf{d}$, the same veins appear collapsed
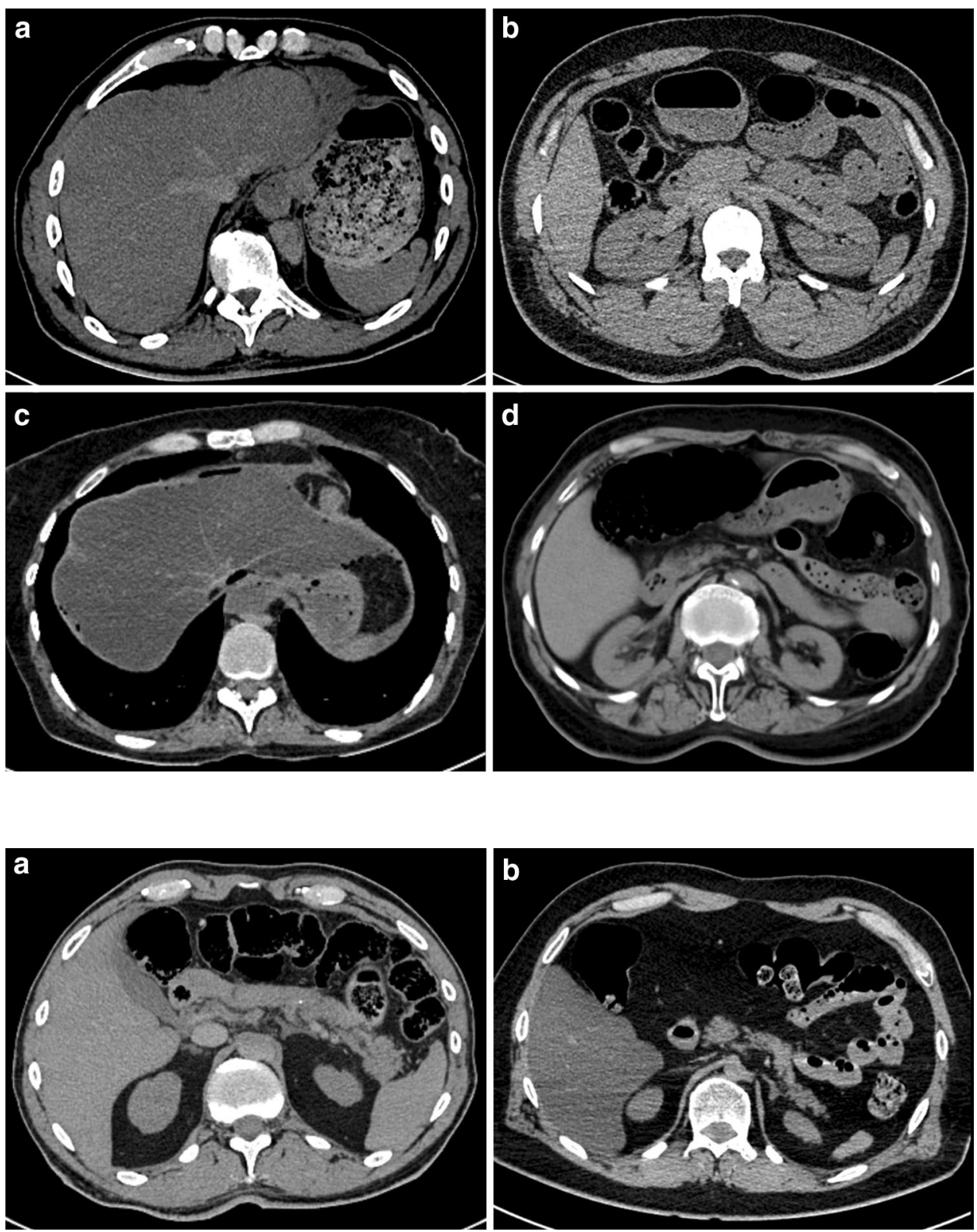

Fig. 5 The CT axial images at the infrahepatic level are referred to a case of the study group (a) and to a case of the control group (b). Note the distension of the IVC in a and the almost complete collapse in $\mathbf{b}$
All the 11 subjects of the control group did not show the presence of FHS (Fig. 2), compression of the coronary sinus and/or of the pulmonary trunk (Fig. 3), distension of the hepatic and/or renal veins (Fig. 4) and periportal oedema. A complete collapse of the SVC was never observed, and the IVC in the infrahepatic tract appeared almost completely collapsed in 10/11 cases (Fig. 5).

\section{Discussion}

The post-mortem evidence of HP and the attribution of the correct relevance of this finding in causing death is challenging for the forensic pathologist, because often clinical/instrumental data are not available to demonstrate the occurrence of a PT against other coexistent heart damage or pathologies that can justify the death.

Post-mortem imaging techniques have been widely introduced in forensic investigations of many institutions all over the word [26-28]. Nevertheless, few studies in the forensic literature investigated on PMCT imaging in deaths with autopsy evidence of HP [18-24].

Huang et al. [18] described PMCT findings in a case of death due to traumatic PT. In this case, PMCT imaging showed a serial rib fractures, a transverse fracture of the sternum, HP and dilated SVC and IVC, as well as bilaterally distended renal veins. Based on these results, the authors attributed the death to PT.

Shiotani et al. [20] analysed PMCT appearances of HP in deceased acute aortic dissection patients. The authors 
Table 2 Results of PMCT imaging analysis in the control group

\begin{tabular}{|c|c|c|c|c|c|c|c|c|}
\hline Case no. & FHS & $\uparrow \varnothing \mathrm{SVC}$ & $\uparrow \varnothing \mathrm{IVC}$ & $\uparrow \varnothing$ Hepatic veins & $\uparrow \varnothing$ Renal veins & $\downarrow \varnothing$ Coronary sinus & $\downarrow \varnothing$ Pulmonary trunk & Periportal oedema \\
\hline 1. & - & \pm & - & - & - & - & - & - \\
\hline 2. & - & \pm & - & - & - & - & - & - \\
\hline 3. & - & \pm & - & - & - & - & - & - \\
\hline 4. & - & \pm & - & - & - & - & - & - \\
\hline 5. & - & \pm & - & - & - & - & - & - \\
\hline 6. & - & \pm & - & - & - & - & - & - \\
\hline 7. & - & \pm & - & - & - & - & - & - \\
\hline 8. & - & \pm & \pm & - & - & - & - & - \\
\hline 9. & - & \pm & - & - & - & - & - & - \\
\hline 10. & - & \pm & - & - & - & - & - & - \\
\hline 11. & - & \pm & - & - & - & - & - & - \\
\hline
\end{tabular}

FHS flattened heart sign, $S V C$ superior vena cava, IVC inferior vena cava

- Finding absent, + finding markedly present, \pm finding not markedly present

described a double-concentric stratification of intrapericardial blood with the densest ring on the epicardial surface in $18 / 30$ cases. The authors explained this particular imaging appearance of HP, named HAH, as due to the stratification of blood cells on the epicardial surface (high-density ring) and of serum along the pericardium (low-density outer ring).

Huang and Shiotani $[18,20]$ did not carry out autopsy, but the assumed causes of death were based on comprehensive health records and information on the medical history.

Recently, Filograna et al. [23, 24] in a research study about PMCT imaging in cases with HP and in a case report about an HP due to ruptured aortic dissection suggested the possibility to use PMCT imaging results to retrospectively assess the diagnosis of PT. In these two papers, the authors advice the opportunity to consider both the PMCT appearance of HP, as described by Shiotani [20], but also the in vivo $\mathrm{CT}$ findings reported in the radiological clinical literature as suggestive of PT, to support the "post-mortem" diagnosis of fatal HP.

In line with this suggestion, this work investigates on the possibility to define a forensic reference for the post-mortem diagnosis of PT due to HP based on PMCT.

Although the clinical literature does not describe HAH sign [20], it was observed in PMCT images of 13/14 cases of the study group.

According to the previous studies [20, 23, 24], we attribute the presence of this double-concentric stratification to fibrination induced on the blood cells in contact with the epicardial surface by the beating movements of the heart originating the densest inner ring, and to defibrinated plasma, constituting the low-density outer ring. Thus, finding an HAH in cases with HP might mean that the heart was beating at the moment of the intrapericardial bleeding and that it maintained his mechanical function for a while after the bleeding occurred. About the vitality of the HAH, Filograna et al. [24] suggested that post-mortem MR (PMMR) might provide more insights because of its superiority in displaying the internal "structure" of haemorrhages and clots. According to Jackowski et al. [29], in fact, a vital clot should show a homogeneous intensity on PMMR and should be adherent to the walls of the vascular cavity. An inferior sensitivity of PMCT in the detection of HAH, with respect to PMMR, could be also the explanation of the absent detection of HAH in the case no. 12, where the blood in the pericardial sack showed fluid-fluid stratification in PMCT images. Nevertheless, PMMR was not performed in this case.

According to Filograna et al. [23], next to the PMCT appearances of HP, other clinical CT findings have to be considered in the assessment of the diagnosis of PT on post-mortem imaging. In this regard, the present study demonstrates firstly that most of the in vivo CT findings proposed in clinical literature as highly suggestive of an imminent PT might be detected also on images acquired in the post-mortem period. Secondly, this work shows that some PMCT findings prove the diagnosis of PT (i.e. fatal HP).

A number of CT findings that are critical to establish in vivo the diagnosis of an imminent PT (i.e. FHS, compression of the coronary sinus and of the pulmonary trunk, distension of the hepatic and renal veins and periportal oedema) were present exclusively in the 14 cases of PT. Moreover, FHS and compression of the coronary sinus and/ or of the pulmonary trunk, even if with variable severity, were observed in all PT cases. Distension of the hepatic and/or renal veins was frequent finding in the study group (12/14 cases), and absent in the control group. Although periportal oedema was observed only in three cases of the study group, it was never identified in the control group. 
Finally, while SVC was never collapsed in both groups, the IVC in the infrahepatic tract appeared to be collapsed only in 5/14 cases of fatal HP, against the 10/11 cases of the control group. This means that also the distension of IVC can be considered a frequent finding in PT deaths.

Nevertheless, if the particular setting of the present analysis is conceived, some considerations have to be taken. A progressive reduction of the diameters of the circulatory system, especially the aorta, on PMCT scans was pointed out by Ishikawa [25], and a distension of cardiac chambers was described by Shiotani [30]. These phenomena may have an influence on the evaluation of both arterious and venous structures and also heart chambers, by modifying their diameters in post-mortem imaging. Nevertheless, the absence of many findings suggestive of PT in the control group (i.e. FHS, compression of the coronary sinus and of the pulmonary trunk, distension of the hepatic and renal veins) related to circulatory system distension/compression might suggest the effectiveness of this data, also in the post-mortem period. On the other hand, personal experience shows that the distension of the abdominal venous system is a common finding in cases of death due to cardiac diseases.

A spontaneous question is whether these same PMCT findings could be detected also in cases with non-fatal HP. Other studies are needed to solve this doubt. Nevertheless, we agree with Restrepo [15], when he states that, although the reported clinical findings, when isolated cannot be considered specific for the diagnosis of an imminent PT, their simultaneous detection can strongly suggest this diagnosis, particularly in the presence of a large pericardial effusion. Similarly in the post-mortem setting, the previous study of Filograna et al. [24] suggests that although HAH sign and FHS could be individually detected in both fatal and non-fatal cases of HP, the association of both signs can be observed only in deaths due to PT.

Regarding the limitations of the study, the limited number of cases should be considered the major one. A large study population might better sustain our hypotheses.

In conclusion, this work demonstrates the potentials of post-mortem imaging in the assessment of the diagnosis of PT. Particularly, the study shows that in cases with HP and an intact pericardium, the diagnosis of death due to PT could be supported by the co-presence of HAH, FHS, compression of the coronary sinus and/or pulmonary trunk, distension of hepatic and renal veins. These results demonstrate that PMCT imaging may add supplementary data to autopsy. We are conscious of the possible danger of misinterpretation of post-mortem imaging. Nevertheless, our results suggest also that PMCT might be considered superior or even substitutive to autopsy, the actual gold standard, in those cases with HP and an intact pericardium where perimortem clinical or instrumental data are not available.
Other important benefit of using PMCT with respect to autopsy is the fact that it is a minimally invasive, reproducible and operator-safe technique, which could be used also as a screening method; this is even truer considering the decline of autopsy rates over the last years. We are aware that it is not often possible to perform a PMCT examination on all deaths, especially in those institutions where a dedicated CT scanner is not available for forensic purposes. Nevertheless, we suggest to perform a PMCT examination on all sudden deaths where a cardiac origin is suspected.

Further studies are needed to verify if the proposed model for the diagnosis of PT can be applied in cases where the pericardium is not intact and/or other pathological conditions may have caused or concurred to cause the death.

Nevertheless, this study confirms and demonstrates the hypothesis of using post-mortem evidence as a mirror of a dynamic in vivo clinical condition, such as PT, by means of PMCT [23, 24].

Conflict of interest The authors declare no conflict of interest.

Ethical standards This article does not contain any study with animals performed by any of the authors.

\section{References}

1. Saukko P, Knight B (1996) Chest and abdominal injuries. In: Saukko P, Knight B (eds) Forensic pathology. Arnold, London, p 228

2. Eisenmenger W (2004) Spitze, scharfe und halbscharfe Gewalt. In: Brinkmann B, Madea B (eds) Handbuch Gerichtliche Medizin, vol 1. Springer, Berlin, p 576

3. Klein AL, Abbara S, Agler DA, Appleton CP, Asher CR, Hoit B, Hung J, Garcia MJ, Kronzon I, Oh JK, Rodriguez ER, Schaff HV, Schoenhagen P, Tan CD, White RD (2013) American Society of Echocardiography clinical recommendations for multimodality cardiovascular imaging of patients with pericardial disease: endorsed by the Society for Cardiovascular Magnetic Resonance and Society of Cardiovascular Computed Tomography. J Am Soc Echocardiogr 26:965-1012

4. Wann S, Passen E (2007) Echocardiography in pericardial disease. J Am Soc Echocardiogr 21:7-13

5. Oyama N, Oyama N, Komuro K, Nambu T, Manning WJ, Miyasaka K (2004) Computed tomography and magnetic resonance imaging of the pericardium: anatomy and pathology. Magn Reson Med Sci 3:145-152

6. Killeen KL, Poletti PA, Shanmuganathan K, Mirvis SE (1999) CT diagnosis of cardiac and pericardial injuries. Emerg Radiol 6:339-344

7. Krejci CS, Blackmore CC, Nathens A (2000) Hemopericardium: an emergent finding in a case of blunt cardiac injury. AJR Am J Roentgenol 175:250

8. Harries SR, Fox BM, Roobottom CA (1998) Azygos reflux: a CT sign of cardiac tamponade. Clin Radiol 53:702-704

9. Rotondo A, Scialpi M, Catalano O, Strada A, Grassi R, Angelelli G (1999) Periportal lymphatic distension resulting from cardiac tamponade: CT findings and clinical-pathologic correlation. Emerg Radiol 6:85-93 
10. Hernandez-Luyando L, Calvo J, de las Gonzalez Heras E, de la Puente H, Lopez C (1996) Tension pericardial collections: sign of "flattened heart" in CT. Eur J Radiol 23:250-252

11. Steiner MA, Marshall JJ (2000) Coronary sinus compression as a sign of cardiac tamponade. Catheter Cardiovasc Interv 49:455-458

12. Doppman JL, Rienmuller R, Lissner J et al (1981) Computed tomography in constrictive pericardial disease. J Comput Assist Tomogr 5:1-11

13. Frantz KM, Fishman EK (1992) Hemopericardium leading to cardiac tamponade in the traumatized pediatric patient: discovery by CT. Clin Imaging 16:180-182

14. Chong HH, Plotnick GD (1995) Pericardial effusion and tamponade: evaluation, imaging modalities, and management. Compr Ther 21:378-385

15. Restrepo CS, Lemos DF, Lemos JA, Velasquez E, Diethelm L, Ovella TA, Martinez S, Carrillo J, Moncada R, Klein JS (2007) Radiographics 27:1595-1610

16. Karger B, Niemeyer J, Brinkmann B (1999) Physical activity following fatal injury from sharp pointed weapons. Int J Legal Med 112:188-191

17. Moritz AR (1942) The pathology of trauma. Lea \& Febiger, Philadelphia

18. Huang P, Wan L, Qin Z, Zhang J, Liu N, Zou D, Chen Y (2012) Post-mortem MSCT diagnosis of acute pericardial tamponade caused by blunt trauma to the chest in a motor-vehicle collision. Rom J Leg Med 20:117-122

19. Filograna L, Hatch G, Ruder T, Ross SG, Bolliger SA, Thali MJ (2013) The role of post-mortem imaging in a case of sudden death due to ascending aorta aneurysm rupture. Forensic Sci Int 228:e76-e80

20. Shiotani S, Watanabe K, Kohno M, Ohashi N, Yamazaki K, Nakayama H (2004) Postmortem computed tomographic (PMCT) findings of pericardial effusion due to acute aortic dissection. Radiat Med 22:405-407
21. Ebert LC, Ampanozi G, Ruder TD, Hatch G, Thali MJ, Germerott $\mathrm{T}$ (2012) CT based volume measurement and estimation in cases of pericardial effusion. J Forensic Leg Med 19:126-131

22. Ebert LC, Schön CA, Ruder TD, Thali MJ, Hatch GM (2012) Fatal left ventricular rupture and pericardial tamponade following a horse kick to the chest. Am J Forensic Med Pathol 33:167-169

23. Filograna L, Flach PM, Bolliger SA, Thali MJ (2014) The role of post-mortem CT (PMCT) imaging in the diagnosis of pericardial tamponade due to hemopericardium: a case report. Leg Med 16:150-153

24. Filograna L, Thali MJ, Marchetti D (2014) Forensic relevance of post-mortem CT imaging of the hemopericardium in determining the cause of death. Leg Med 16:247-251

25. Ishikawa N, Nishida A, Miyamori D, Kubo T, Ikegaya H (2013) Estimation of postmortem time based on aorta narrowing in CT imaging. J Forensic Leg Med 2010:75-77

26. Rutty GN, Morgan B, O'Donnell C, Leth PM, Thali M (2008) Forensic institutes across the world place CT or MR scanners or both into their mortuaries. J Trauma 63:493-494

27. Tartaglione T, Filograna L, Roiati S, Guglielmi G, Colosimo C, Bonomo L (2012) Importance of 3D-CT imaging in single-bullet cranioencephalic gunshot wounds. Radiol med 117:461-470

28. Ambrosetti MC, Barbiani C, El-Dalati G, Pellini E, Raniero D, De Salvia A, Pozzi Mucelli R (2013) Virtual autopsy using multislice computed tomography in forensic medical diagnosis of drowning. Radiol Med 118:679-687

29. Jackowski C, Thali M, Aghayev E, Yen K, Sonnenschein M, Zwygart K, Dirnhofer R, Vock P (2006) Postmortem imaging of blood and its characteristics using MSCT and MRI. Int J Legal Med 120:233-240

30. Shiotani S, Kohno M, Ohashi N, Yamazaki K, Nakayama H, Watanabe K, Itai Y (2003) Dilatation of the heart on postmortem computed tomography (PMCT): comparison with live CT. Radiat Med 21:29-35 